\title{
Estudio psicométrico preliminar de la escala de motivos de uso de videojuegos e-MUV
}

\author{
Fco. Javier López Fernández \\ frlopez@uji.es \\ Jordi Ortet Walker \\ jortet@uji.es \\ Sígrid Gallego Moya \\ sigridgallego@gmail.com \\ Generós Ortet Fabregat \\ ortet@uji.es
}

\section{Resumen}

Introducción: Los videojuegos son una de las actividades de ocio más importantes, especialmente entre la juventud. Durante los últimos años, la preocupación por su uso problemático ha crecido hasta ser considerada una adicción en la actualidad. Una de las diferencias individuales que podrían explicar el uso regular y adictivo de los videojuegos son los motivos. Así, el objetivo del estudio es desarrollar una escala de motivos de uso de videojuegos. Metodología: A través de una revisión en la literatura, se seleccionaron los motivos más frecuentemente encontrados y se testaron sobre una muestra de 140 jóvenes jugadores (79 hombres, media de edad 20,71 años; DT =3,55). También se evaluaron sus horas semanales dedicadas y sus niveles de adicción a los videojuegos (IGD-20) con el fin de asociar estas variables con los motivos encontrados mediante análisis de regresión lineal.

Resultados: Nuestra escala, la escala de motivos de uso de videojuegos (eMUV), estuvo compuesta de 8 motivos formada por 4 ítems cada uno: inmersión, customización, gratificación violenta, afrontamiento, diversión, reto cognitivo, competición e interacción social. El motivo de afrontamiento se asoció principalmente con la adicción a los videojuegos, mientras que la interacción social fue fuertemente relacionada con la cantidad de horas de juego.

Conclusión: La e-MUV presenta índices de fiabilidad y validez adecuados. Por lo tanto, podría ser utilizada para entender mejor las conductas relacionadas con los videojuegos.

Palabras clave: motivos, adicción a los videojuegos, uso de videojuegos, afrontamiento. 


\begin{abstract}
Introduction: Video games are one of the most important leisure activities, especially among youths. During the last years, the concern about its problematic use has grown to be considered an addiction nowadays. One of the individual differences that could explain regular video game use and video game addiction are motives. Therefore, the aim of this study is to develop a scale of motives in video game use.

Method: Through a literature review, the most frequent motives found in research were selected and examined on a sample of 140 young players (79 men, mean age 20.71 years old; $S D=3.55$ ). Furthermore, their gaming hours weekly and their levels of video game addiction (IGD-20) were measured in order to associate these variables with motives found by linear regression analyses.

Results: Our scale, escala de motivos de uso de videojuegos (e-MUV), was composed of 8 motives with 4 items for each one: immersion, customization, violent gratification, coping, enjoyment, cognitive challenge, competition and social interaction. Coping motive was principally associated with video game addiction, whereas social interaction was strongly related to higher gaming hours. Conclusion: The e-MUV shows suitable index of reliability and validity. Thus, it could be useful for understanding better video game-related behaviors.
\end{abstract}

Keywords: motives, video game addiction, video game use, coping.

\title{
Introducción
}

La industria del videojuego en España constituye la primera opción de ocio audiovisual, superando con creces en la actualidad a los ingresos económicos de la industria del cine y de la música. De esta manera, 15 millones de españoles juegan a videojuegos regularmene con una frecuencia media de 6,2 horas semanales. Así, la mayor frecuencia de uso se encuentra en la infancia y la juventud. Por ejemplo, en el rango de edad entre los 15 y 24 años, un $68 \%$ juega a videjuegos regularmente (AEVI 2016).

En los últimos años, la preocupación por las consecuencias del uso abusivo de videojuegos se ha visto incrementada. Así, la última versión del Manual Diagnóstico y Estadístico de Trastornos Mentales (DSM-V, según sus siglas en inglés) propone el trastorno de juego por Internet como una potencial adicción conductual (APA 2013). El trastorno se centra en los videojuegos online aunque los videojuegos offline podrían verse también incorporados. En este sentido, recientemente, la Organización Mundial de la Salud (OMS) ha incluido la adicción a los videojuegos en la clasificación internacional de enfermades (ICD-11, según sus siglas en inglés) (Aarseth et al. 2017). De este modo, tanto las adicciones a sustancias como las adicciones conductuales compartirían un similar criterio diagnóstico, como la saliencia, la modificación del estado de ánimo, la tolerancia, la abstinencia (psicológica), el conflicto con aspectos vitales o la recaída (Griffiths 2005; Petry, Rehbein, Ko y O'Brien 2015). Se estima que la prevalencia mundial en la adicción a los videojuegos se encuentra entre el $1 \mathrm{y}$ el $9 \%$ de la población, dependiendo esta variabilidad de grupos de edad y diferencias socioculturales (Gentile et al. 2017). Así, existe una mayor prevalencia del trastorno en edades jóvenes y en el género varón (Petry et al. 2015; Mihara e Higuchi 2017).

De este modo, diversas diferencias individuales deberían explicar parte de la variabilidad observada en el uso regular y problemático de los videojuegos, como la 
personalidad (por ejemplo, Mihara e Higuchi 2017) o los motivos de uso. En este sentido, en la investigación podemos observar la relevancia del estudio de los motivos para explicar el uso y el abuso en las adicciones como en el alcohol (Mezquita et al. 2016), el cannabis (Mezquita, Ruiz-Valero, Martínez Gómez, Ibáñez y Ortet 2018) o el juego de apuestas (Stewart y Zack 2008). Particularmente, el estudio del papel de los motivos con respecto a los usos de videojuegos se ha realizado desde distintos enfoques teóricos, como desde la teoría del uso y la gratificación (Sherry, Lucas, Greenberg y Lachlan 2006), la teoría de la autodeteminación (Ryan, Rigby y Przybylski 2006) o la teoría de las necesidades de la psicología humanista (Wan y Chiou 2006).

A pesar del gran número existente de estudios que evalúan el papel de los motivos en las conductas relacionadas con los videojuegos, distintas limitaciones son encontradas en la investigación que nos incitan a desarrollar y validar una nueva escala de motivos en el uso de videojuegos, como un etiquetado distinto para motivos similares a través de diferentes instrumentos, la focalización de las escalas en videojuegos específicos o la escasez de intrumentos que midan motivos en lengua castellana. Así, el objetivo del presente estudio es desarrollar y validar una nueva escala de motivos de uso de videojuegos que, a través de la revisión de la literatura, pueda unificar etiquetados distintos de motivos similares y que, además, mediante la generalización de la redacción de los ítems, pueda ser utilizada de manera universal independientemente del tipo de videojuegos que se use.

\section{Método}

\section{Participantes}

La muestra estaba compuesta por 140 participantes (79 hombres) con una media de edad de 20,71 años (DT =3,55). Siendo la media de edad en hombres 20,85 $(D T=3,70)$ y en mujeres 20,54 (DT = 3,37). 64 fueron voluntarios del evento UJI Game Experience del año 2017, mientras que el resto eran estudiantes del grado de psicología de la Universitat Jaume I.

\section{Procedimiento}

Mediante una revisión de la literatura de un grupo de expertos acerca de las escalas de motivos en el uso de videojuegos, en la que se examinaron 13 escalas distintas, desarrollamos una escala a través de la cual se evaluaron los motivos más recurrentemente encontrados en la investigación. Durante el año 2017, los participantes pudieron contestar a los cuestionarios del estudio de manera online. Estos fueron informados de los objetivos de la investigación, además de la confidencialidad de la misma.

\section{Instrumentos}

La escala de motivos de uso de videojuegos (e-MUV), desarrollada en el presente estudio, estuvo compuesta por 62 ítems de respuesta tipo Likert de 5 puntos (de $1=$ «totalmente en desacuerdo» hasta $5=$ «totalmente de acuerdo»). Esta fue empleada para testar los siguientes 12 motivos: arousal (por ejemplo, «son excitantes y emocionantes»), diversión (por ejemplo, «me entretienen»), social (por ejemplo, «conozco a otras personas»), afrontamiento-escape (por ejemplo, «me ayudan a olvidar problemas del día a día»), catarsis de la violencia (por ejemplo, «me ayuda a canalizar mi agresividad»), recompensa violenta (por ejemplo, «disfruto destrozando 
cosas en el juego»), fantasía (por ejemplo, «me gusta sentirme parte de una historia»), mejora de habilidades (por ejemplo, «agudizan mis sentidos»), customización-creación (por ejemplo, «disfruto diseñando cosas en el juego»), competición (por ejemplo, «me gusta ganar a otros jugadores»), logro (por ejemplo, «porque me siento poderoso en el juego») y conformidad (por ejemplo, «para no sentirme excluído»).

La versión española del Internet Gaming Disorder-20 (IGD-20) test (Fuster, Carbonell, Pontes y Griffiths 2016) fue utilizada para evaluar la adicción a los videojuegos. La escala consta de 20 ítems de respuesta tipo Likert de 5 puntos (de $1=$ «totalmente en desacuerdo» hasta $5=$ «totalmente de acuerdo») y mostró un alfa de Cronbach de 0,88 .

Finalmente, fueron también evaluadas las horas de juego semanales.

\section{Análisis}

El software estadístico SPSS versión 21 fue empleado para realizar el análisis factorial exploratorio de los motivos testados y sus respectivas pruebas de fiabilidad, la matriz de correlaciones entre las variables del estudio y análisis de regresión lineal múltiple para ver el efecto de los motivos, como variables independientes, sobre las variables dependientes de las horas semanales de juego y la adicción, controlando por género y edad.

\section{Resultados}

La muestra presentó una gran variabiliddad en las horas de juego semanales. Así, la media de horas de juego fue de 11,93 y la desviación típica de 12,90 horas.

El análisis factorial mostró la existencia de 8 dimensiones de motivos (inmersión, customización, gratificación violenta, afrontamiento, diversión, reto cognitivo, competición e interacción) de las cuales seleccionamos los 4 ítems que reflejaban una mayor carga factorial. De esta manera, la escala se redujo a 32 ítems que mostraban unos índices de fiabilidad y validez adecuados (véase la tabla 1).

La matriz de correlaciones (véase la tabla 2) reflejó que los hombres mostraban mayores niveles en las motivaciones de interacción social $(r=-0,45 ; p<0,01)$, gratificación violenta $(r=-0,42 ; p<0,01)$ y competición $(r=-0,37 ; p<0,01)$, además de reportar más horas de juego $(r=-0,18 ; p<0,01)$ y niveles de adicción $(r=-0,36$; $p<0,01)$. Por otra parte, las mujeres reportaron mayor nivel de motivación en customización $(r=0,21 ; p<0,05)$. Una mayor edad estuvo asociada a mayores motivaciones de reto cognitivo, inmersión y diversión. La cantidad de horas de juego estuvo relacionada con las motivaciones de interacción social y diversión. Finalmente, la adicción se vinculó a motivos de gratificación violenta, afrontamiento, competición e interacción social.

Los análisis de regresión lineal (véase la tabla 3 ) mostraron que, principalmente, los motivos de interacción social $(\beta=0,42 ; p<0,001)$ predecían una mayor cantidad de horas de juego semanal, además de ser hombres $(\beta=-0,18$; $p<0,05)$. Por otra parte, las puntuaciones en adicción a los videojuegos fueron asociadas a los motivos de afrontamiento $(\beta=0,39 ; p<0,001)$, gratificación violenta $(\beta=0,27 ; p<0,001)$ y al género varón $(\beta=-0,36 ; p<0,001)$. También se encontró una pequeña relación inversa entre la adicción y los motivos de customización $(\beta=-0,19 ; p<0,05)$. 
Tabla 1

Análisis factorial y alfas de Cronbach de los motivos obtenidos

\begin{tabular}{|c|c|c|c|}
\hline Ítem & $\begin{array}{c}\text { Carga } \\
\text { factorial }\end{array}$ & Ítem & $\begin{array}{c}\text { Carga } \\
\text { factorial }\end{array}$ \\
\hline Inmersión & Alfa $=0,88$ & Diversión & Alfa $=0,85$ \\
\hline $\begin{array}{l}\text { Me siento inmerso en un mundo } \\
\text { fantástico/fictio }\end{array}$ & 0,55 & Disfruto jugando & 0,75 \\
\hline $\begin{array}{l}\text { Me gusta sentirme parte de una } \\
\text { historia }\end{array}$ & 0,50 & Me entretienen & 0,74 \\
\hline $\begin{array}{l}\text { Disfruto metiéndome en la piel de } \\
\text { un personaje en cada juego }\end{array}$ & 0,41 & Me lo paso bien & 0,70 \\
\hline $\begin{array}{l}\text { Me gusta explorar el mundo del } \\
\text { juego para descubrir cosas } \\
\text { nuevas }\end{array}$ & 0,34 & Es divertido & 0,64 \\
\hline Customización & Alfa $=0,88$ & Reto cognitivo & Alfa $=0,79$ \\
\hline $\begin{array}{l}\text { Disfruto diseñando cosas en el } \\
\text { juego }\end{array}$ & 0,89 & Me suponen un reto mental & 0,78 \\
\hline $\begin{array}{l}\text { Me gusta crear cosas en el juego, } \\
\text { como casas u otras } \\
\text { construcciones }\end{array}$ & 0,87 & Me hacen más inteligente & 0,67 \\
\hline $\begin{array}{l}\text { En el juego me gusta utilizar } \\
\text { distintos elementos para crear } \\
\text { cosas nuevas }\end{array}$ & 0,72 & $\begin{array}{l}\text { Me hacen pensar / calentarme la } \\
\text { cabeza }\end{array}$ & 0,65 \\
\hline $\begin{array}{l}\text { Me gusta diseñar o personalizar } \\
\text { la apariencia de mis personajes }\end{array}$ & 0,69 & Mejoran mis habilidades & 0,42 \\
\hline Gratificación violenta & Alfa $=0,88$ & Competición & Alfa $=0,81$ \\
\hline $\begin{array}{l}\text { Me gusta la violencia en el juego, } \\
\text { cuanto más, mejor }\end{array}$ & 0,88 & Me gusta ganar & 0,70 \\
\hline $\begin{array}{l}\text { Disfruto de las peleas y luchas } \\
\text { violentas en el juego }\end{array}$ & 0,87 & $\begin{array}{l}\text { Me gusta demostrar que soy } \\
\text { mejor que otros jugadores }\end{array}$ & 0,69 \\
\hline $\begin{array}{l}\text { En el juego es divertido disparar a } \\
\text { alguien en la cabeza }\end{array}$ & 0,68 & $\begin{array}{l}\text { Me gusta provocar o picar a } \\
\text { otros jugadores }\end{array}$ & 0,63 \\
\hline $\begin{array}{l}\text { Disfruto destrozando cosas en el } \\
\text { juego }\end{array}$ & 0,66 & Disfruto compitiendo con otros & 0,56 \\
\hline Afrontamiento & Alfa $=0,84$ & Interacción social & Alfa $=0,89$ \\
\hline Olvido mis preocupaciones & 0,82 & Hago nuevos amigos & 0,85 \\
\hline $\begin{array}{l}\text { Me permiten escapar del mundo } \\
\text { real }\end{array}$ & 0,71 & $\begin{array}{l}\text { Mediante el juego estoy en } \\
\text { contacto con mis amigos }\end{array}$ & 0,83 \\
\hline $\begin{array}{l}\text { Me ayudan a mejorar mi estado } \\
\text { de ánimo }\end{array}$ & 0,54 & $\begin{array}{l}\text { Me gusta jugar con gente, online } \\
\text { o en la misma habitación }\end{array}$ & 0,69 \\
\hline $\begin{array}{l}\text { Me permite sentirme mejor } \\
\text { cuando estoy frustrado }\end{array}$ & 0,49 & Disfruto jugando en grupo & 0,68 \\
\hline
\end{tabular}


Tabla 2

Matriz de correlaciones de las variables del estudio

\begin{tabular}{lllll}
\hline & 1 & 2 & 3 & 4 \\
\hline 1. Sexo & - & & & \\
2. Edad & $-0,04$ & - & & \\
3. Horas de juego & $-0,18^{* *}$ & $-0,02$ & - & \\
4. Adicción & $-0,36^{* *}$ & $-0,07$ & $0,38^{* *}$ & - \\
Inmersión & $-0,12$ & $0,19^{*}$ & 0,11 & 0,08 \\
Customización & $0,21^{*}$ & 0,14 & 0,04 & $-0,10$ \\
Gratificación violenta & $-0,42^{* *}$ & $-0,12$ & 0,14 & $0,44^{* *}$ \\
Afrontamiento & 0,02 & 0,02 & 0,15 & $0,38^{* *}$ \\
Diversión & 0,14 & $0,17^{*}$ & $0,24^{* *}$ & 0,12 \\
Reto cognitivo & $-0,10$ & $0,25^{* *}$ & 0,03 & 0,09 \\
Competición & $-0,37^{* *}$ & $-0,08$ & 0,12 & $0,32^{\star *}$ \\
Interacción social & $-0,45^{* *}$ & $-0,02$ & $0,40^{* *}$ & $0,28^{* *}$ \\
\hline
\end{tabular}

Nota: ${ }^{*} \mathrm{p}<0,05 ;{ }^{* *} \mathrm{p}<0,01$

género: $1=$ hombres, 2 = mujeres

Tabla 3

Análisis de regresión

\begin{tabular}{lll}
\hline & Horas de juego & Adicción \\
\hline 1 & & \\
Sexo & $-0,18^{*}$ & $-0,36^{* * *}$ \\
Edad & $-0,03$ & $-0,09$ \\
$\Delta R^{2}$ & 0,03 & $0,14^{* * *}$ \\
\hline 2 Inmersión & & \\
Customización & 0,05 & $-0,02$ \\
Gratificación violenta & $-0,04$ & $-0,19^{*}$ \\
Afrontamiento & 0,05 & $0,27^{* *}$ \\
Diversión & 0,13 & $0,39^{* * *}$ \\
Reto cognitivo & 0,16 & 0,03 \\
Competición & $-0,16$ & $-0,14$ \\
Interacción social & $-0,14$ & 0,08 \\
$\Delta R^{2}$ & $0,42^{* * *}$ & 0,09 \\
$R^{2}$ & $0,19^{* * *}$ & $0,24^{* * *}$ \\
\hline
\end{tabular}

Nota: Coeficientes $\beta$ signficativos: ${ }^{*} p<0,05 ;{ }^{* *} p<0,01 ;{ }^{* * *} p<0,001$

\section{Discusión y conclusiones}

Nuestro trabajo tenía el objetivo de desarrollar y validar una nueva escala de motivos para el uso de videojuegos. Para ello se testaron los motivos más recurrentes encontrados en la investigación. Como resultado, obtuvimos una escala de 8 motivos (inmersión, customización, gratificación violenta, afrontamiento, diversión, reto cognitivo, competición e interacción social) con adecuados índices de fiabilidad y validez. Así, estos factores de motivación obtenidos en el presente estudio son frecuentemente observados en otras investigaciones (por ejemplo, Sherry et al. 2006; Demetrovics et al. 2011).

Nuestros resultados son similares a los de otros estudios basados en las asociaciones entre motivaciones con frecuencia de uso de los videojuegos, así como con su uso adictivo. De esta manera, la interacción social parece ser una motivación clave para 
predecir un mayor tiempo en el uso de videojuegos (Sherry et al. 2006; Greenberg, Sherry, Lachlan, Lucas y Holmstrom 2010). Así, el jugar con amigos o en grupo resultaría más divertido e involucraría un mayor número de horas dedicadas al videojuego. Por otra parte, los motivos de afrontamiento deben ser los más importante a la hora de predecir la adicción a los videojuegos (Kim et al. 2016; Laconi, Pirès y Chabrol 2017). De esta manera, jugar para regular tu estado de ánimo u olvidar tus problemas indicaría mayores problemas con el uso de videojuegos.

Futuros estudios deberían avanzar con la validación de la escala. Por ejemplo, asociando los motivos con los tipos de videojuegos más jugados o con rasgos de personalidad.

Los videojuegos son una de las formas de entretenimiento más populares en nuestros días. Así, una mejor comprensión de las asociaciones de estos con rasgos psicosociales nos ayudaría a prevenir o tratar los problemas que se podrían derivar de su uso patológico.

\section{Referencias bibliográficas}

Aarseth, Espen, Anthony M. Bean, Huub Boonen, Michelle Colder Carras, Mark Coulson, Dimitre Das, Jory Deleuze, Elza Dunkels, Johan Edman, Christopher J. Ferguson... y Maria C. Haagsma. 2017. «Scholars' open debate paper on the World Health Organization ICD-11 Gaming Disorder proposal». Journal of Behavioral Addictions 6(3): 267-270.

American Psychiatric Association. 2013. Diagnostic and statistical manual of mental disorders (5th ed.). Arlington, VA: American Psychiatric Publishing.

Asociación Española de Videojuegos (AEVI). 2016. El anuario del videojuego 2016. Recuperado de: http://www.aevi.org.es/web/wpcontent/uploads/2017/06/ANUARIO_AEVI_2016.pdf [Consultado el 31 de marzo de 2018].

Demetrovics, Zsolt, Róbert Urbán, Katalin Nagygyörgy, Judit Farkas, Dalma Zilahy, Barbara Mervo, Antónia Reindl, Csilla Ágoston, Andrea Kertész y Eszter Harmath. 2011. "Why do you play? The development of the motives for online gaming questionnaire (MOGQ)». Behavior Research Methods 43(3): 814-825.

Fuster, Héctor, Xavier Carbonell, Halley M. Pontes y Mark D. Griffiths. 2016. «Spanish validation of the internet gaming disorder-20 (IGD-20) test». Computers in Human Behavior 56: 215-224.

Gentile, Douglas A., Kira Bailey, Daphne Bavelier, Jeanne Funk Brockmyer, Hilarie Cash, Sarah M. Coyne, Andrew Donan, Donald S. Grnat... y Kimberly Young. 2017. "Internet Gaming Disorder in Children and Adolescents». Pediatrics 140(Supplement 2): S81-S85. http://doi.org/10.1542/peds.2016-1758H

Greenberg, Bradley S., John L. Sherry, Kenneth A. Lachlan, Kristen Lucas y Amanda Holmstrom. 2010. "Orientations to Video Games Among Gender and Age Groups». Simulation y Gaming 41(2): 238-259.

Griffiths, Mark D. 2005. "A 'components' model of addiction within a biopsychosocial framework». Journal of Substance Use 10: 191-197.

Kim, Na Rim, Samuel Suk-Hyun Hwang, Jung-Seok Choi, Dai-Jin Kim, Zsolt Demetrovics, Orsolia Király, Katalin Nagygyörgy, Mark D. Griffiths, So Yeon Hyun, Hyun Chul Youn y Sam-Wook Choi. 2016. "Characteristics and psychiatric symptoms of internet gaming disorder among adults using selfreported DSM-5 criteria». Psychiatry investigation 13(1): 58-66.

Laconi, Stéphanie, Sophie Pirès y Henri Chabrol. 2017. «Internet gaming disorder, motives, game genres and psychopathology». Computers in Human Behavior 75: 652-659. http://doi.org/10.1016/J.CHB.2017.06.012 
Mezquita, Laura, Manuel Ignacio Ibáñez, Jorge Moya-Higueras, Helena Villa, Bárbara Arias, Lourdes Fañanás y Generós Ortet. 2016. «Psychometric properties of drinking motives questionnaire-revised (DMQ-R) in spanish adolescents». European Journal of Psychological Assessment 34: 145-153.

Mezquita, Laura, Lucía Ruiz-Valero, Naiara Martínez Gómez, Manuel Ignacio Ibáñez y Generós Ortet. 2018. «Desarrollo y validación de la versión breve del cuestionario de motivos de consumo de marihuana (MMM SF)». Adicciones. Revista versión online 31(2). Recuperado de: http://www.adicciones.es/index.php/adicciones/article/view/979 [Consultado el 4 de agosto de 2019].

Mihara, Satoko y Sumusu Higuchi. 2017. "Cross-sectional and longitudinal epidemiological studies of internet gaming disorder: A systematic review of the literature». Psychiatry and Clinical Neurosciences 71(7): 425-444.

Petry, Nancy M., Florian Rehbein, Chih-Hung Ko y Charles P. O’Brien. 2015. «Internet gaming disorder in the DSM-5». Current psychiatry reports 17(9): 72.

Ryan, Richard M., C. Scott Rigby y Andrew Przybylski. 2006. "The motivational pull of video games: A self-determination theory approach». Motivation and emotion 30(4): 344-360.

Sherry, John L., Kristen Lucas, Bradley S. Greenberg y Kenneth Lachlan. 2006. «Video game uses and gratifications as predictors of use and game preference». Playing video games: Motives, responses, and consequences 24: 213-224.

Stewart, Sherry H. y Martin Zack. 2008. «Development and psychometric evaluation of a three-dimensional Gambling Motives Questionnaire». Addiction 103(7): 11101117.

Wan, Chin-Sheng. y Wen-Bin Chiou. 2006. «Psychological motives and online games addiction: Atest of flow theory and humanistic needs theory for taiwanese adolescents». CyberPsychology \& Behavior 9(3): 317-324. 